\title{
71. COMPUTER MODELS OF SPIRAL STRUCTURE
}

\author{
F. HOHL \\ NASA Langley Research Center, Hampton, Va., U.S.A.
}

\begin{abstract}
A computer model for isolated disks of stars is used to study the self-consistent motion of large numbers of point masses as they move in the plane of the galactic disk. The Langley Research Center's CDC 6600 computers are used to integrate the equations of motion for systems containing from 50000 to 200000 stars. The results are presented in the form of a motion picture.
\end{abstract}

\section{The Model}

The reported work on computer simulation of disks of stars has so far been confined to infinite doubly periodic systems (Miller and Prendergast, 1968) or to a small numbers of stars (Lindblad, 1960). The model used in the present report simulates the evolution of an isolated disk of stars. Each star in the system moves according to Newton's laws of motion in the self-consistent gravitational field due to the whole system. The motion of the point stars is confined to the plane of the disk. The gravitational potential is calculated on a fixed $N \times N$ array of cells (either $64 \times 64$ or $128 \times$ $128)$ by Fourier transform methods. The model is described in detail by Hohl and Hockney (1969).

The motion of the stars is advanced stepwise in time. One complete cycle for advancing the motion of the system by a small time step consists of the following steps:

(a) The star coordinates are examined to determine the mass density at the center of each of the $N \times N$ array of cells.

(b) From the density distribution over the fixed array of cells, the corresponding gravitational potential at the cell centers is calculated.

(c) The gravitational field at the position of the stars is calculated and is used to advance the position and velocity of the stars by a small time step.

The above three steps represent one cycle and they are repeated until the desired evolution of the system is achieved. The time step is taken sufficiently small and the number of stars sufficiently large such that a decrease in the time step or an increase in the number of stars will not affect the evolution of the system. Results obtained for systems containing 50000 stars and using 200 time steps per rotational period were found to be qualitatively indistinguishable from those for systems with 200000 stars and using 400 time steps per rotational period.

\section{Results}

The first systems investigated were initially balanced, uniformly rotating disks of stars with an initial surface density given by $\sigma(r)=\sigma(0) \cdot\left(1-r^{2} / R_{0}^{21 / 2}\right.$, where $r$ is the radial coordinate and $R_{0}$ is the radius of the disk. As predicted by Toomre (1964), the cold (zero velocity dispersion) disk is found to be unstable. The initial star positions 
are obtained by using a pseudo-random number sequence. Depending on the small perturbations caused by the random initial positions, the cold disk breaks up into three to five smaller subsystems during the first rotation. Adding increasing amounts of initial velocity dispersion has the effect of increasing the smallest size of the star condensations that are formed. A velocity dispersion equal to about $27 \%$ of the circular velocity at the edge of the cold balanced disk stabilizes the disk against breakup and any local condensations. These results are in agreement with the predictions by Toomre (1964). However, the disks were not stable against long wavelength modes and after about two rotations the disks tend to assume a bar-shaped structure. Similar
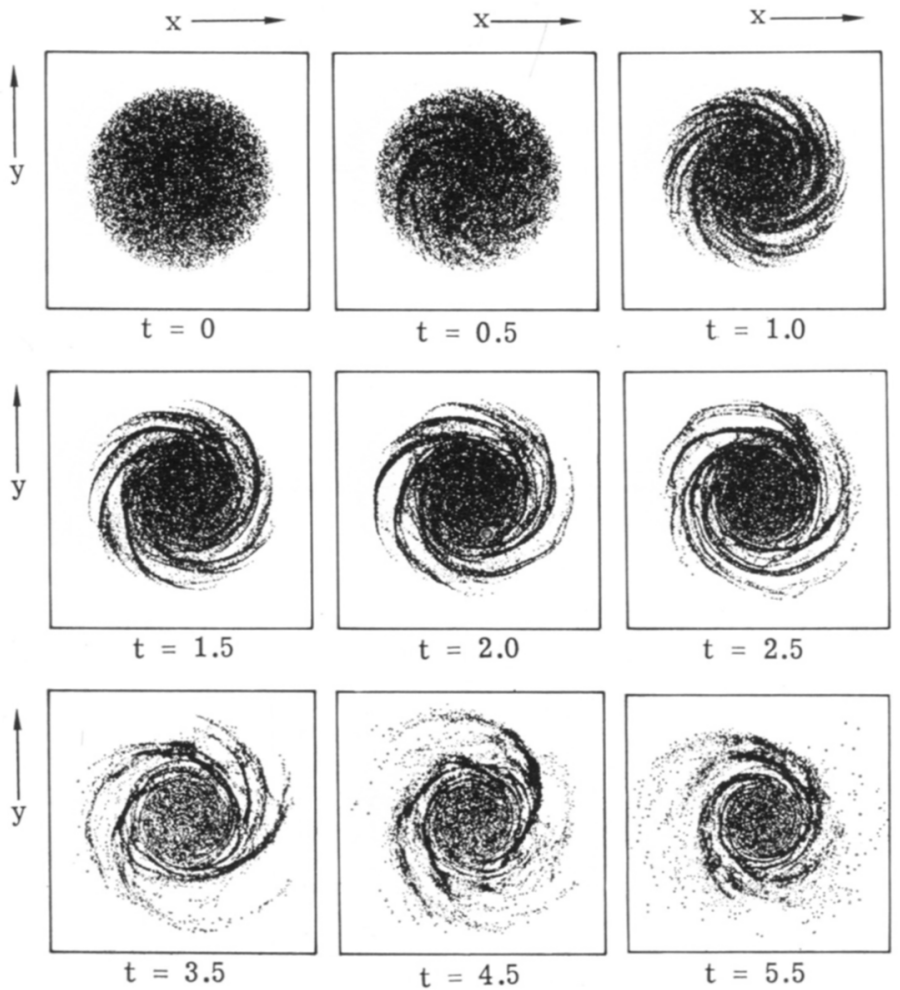

$\mathrm{t}=4.5$
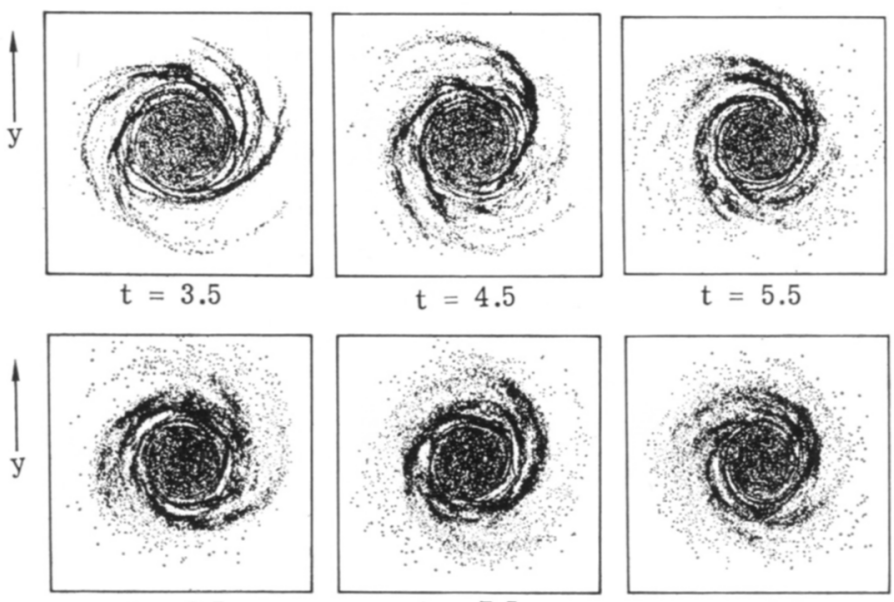

$\mathrm{t}=7.5$

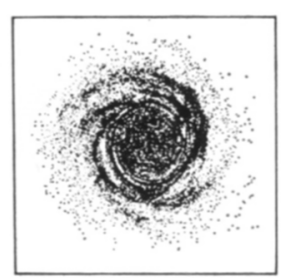

$\mathbf{t}=8.5$

Fig. 1. Evolution of an initially cold balanced disk of stars under the influence of a fixed radial force similar to the Schmidt model. The initial disk radius is $15 \mathrm{kpc}$ and the time is given in rotation periods at a radius of $10 \mathrm{kpc}$. 
results were obtained for disks with a Gaussian density distribution and with a velocity dispersion equal to (or even greater than) the stabilizing velocity dispersion calculated by Toomre (1964). It is found that rather large central condensations of mass (such as an exponential density distribution) are necessary for a stable disk galaxy.

To study the development of spiral structure, the model was modified to include a fixed central force similar to the Schmidt model of the Galaxy. The mass of the stars in the disk was taken to be from 5 to $50 \%$ of the total mass of the Galaxy. The initial distribution of the disk population of stars was a Maclaurin disk with zero radial velocities of the stars and with rotational velocities just sufficient to balance the
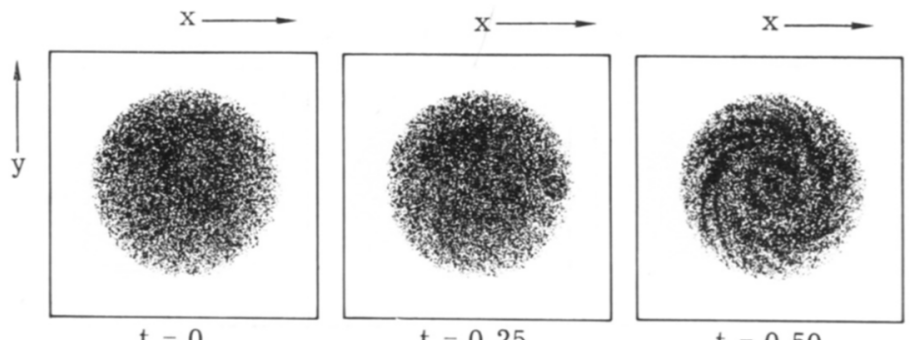

$\mathrm{t}=0.25$

$\mathrm{t}=0.50$

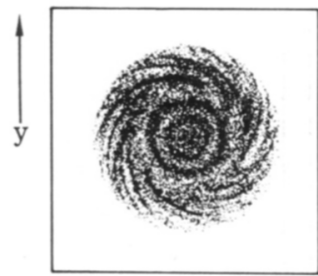

$\mathrm{t}=0.75$

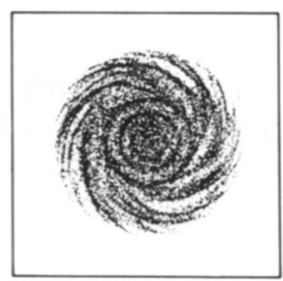

$\mathrm{t}=1.00$

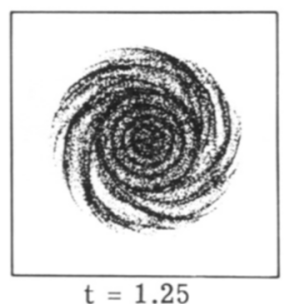

$\mathrm{t}=1.25$

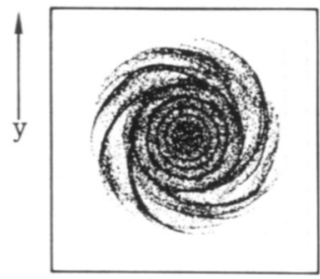

$\mathrm{t}=1.50$

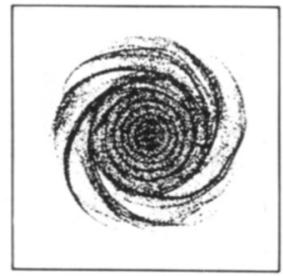

$\mathrm{t}=1.75$
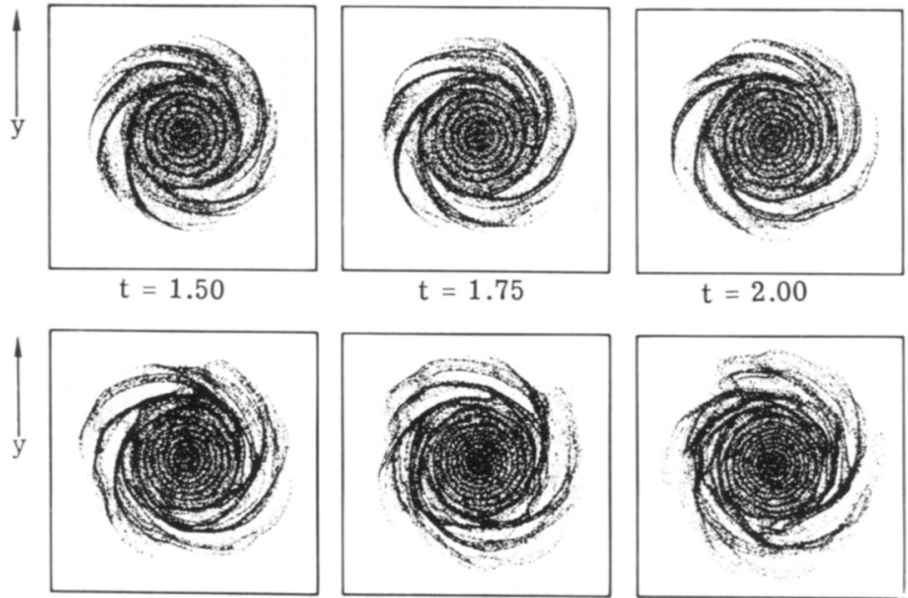

$\mathrm{t}=2.50$

$\mathrm{t}=2.00$

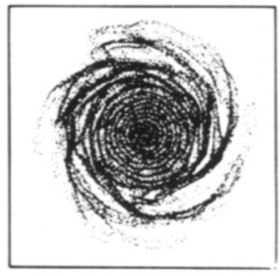

$\mathrm{t}=2.75$

Fig. 2. Evolution of a disk of stars identical to the system shown in Figure 1, except that the initial star positions have received a small perturbation which is proportional to the distance of the star from the center of the disk. 
gravitational attraction. Figure 1 shows the evolution of such a system containing 50000 stars. The disk stars represent $10 \%$ of the total mass of the galaxy and the initial radius of the disk is $15 \mathrm{kpc}$. The time shown is in rotational periods at a radius of 10 kpc. It can be seen that a spiral structure develops and remains even after 8.5 rotations, at which time the calculation was terminated. From a purely kinematical viewpoint, differential rotation should have wound up the spiral structure before 8.5 rotations. In many of the calculations, there appeared to be a tendency to form circular rings of stars in the central region of the disk. To investigate the appearance of the rings more closely, the initial conditions for the system shown in Figure 1 were perturbed by rotating (around the disk center) the initial star positions, about $2^{\circ}$ from their equilibrium positions. The resulting evolution is shown in Figure 2. Note that the circular rings originate at the center of the disk and move outward, resulting in circular density waves. These density waves result purely from the epicyclic motion of the stars and no net mass is carried outward by the density waves. Figure 3 is a close-up of the system at $t=0.75$ and shows in greater detail the structure of the system.

\section{Acknowledgement}

The author would like to thank Professor Alar Toomre for numerous discussions and suggestions.

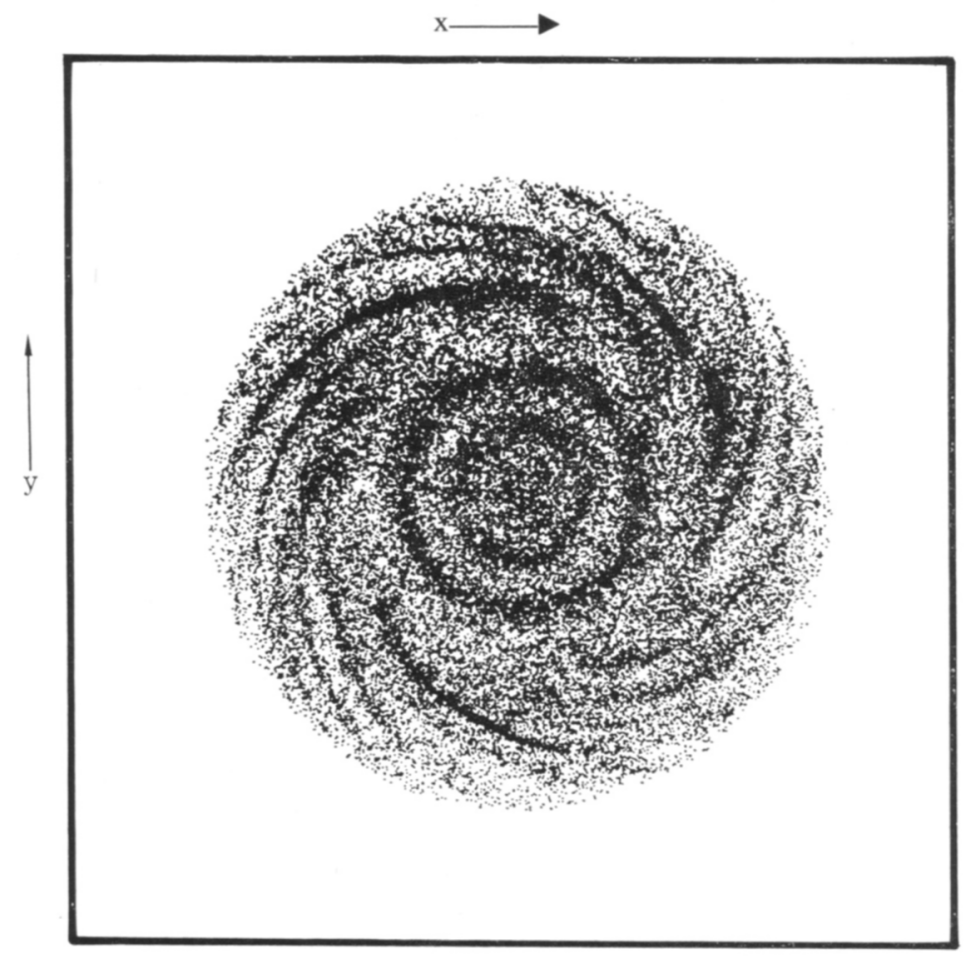

Fig. 3. An enlargement of the system shown in Figure 2 at $t=0.75$. 


\section{References}

Miller, R. H. and Prendergast, K. H.: 1968, Astrophys. J. 151, 699.

Lindblad, P. O.: 1960, Stockholm Obs. Ann. 21, No. 4.

Hohl, F. and Hockney, R. W.: 1969, J. Comput. Phys. 4, 306.

Toomre, A.: 1964, Astrophys. J. 139, 1217. 